JOURNAL OF

FUNCTION SPACES AND APPLICATIONS

Volume 9, Number 2 (2011), 205-215
(C) 2011, Scientific Horizon http://www.jfsa.net

\title{
Fuzzy approximation of an additive functional equation
}

\section{G. Zamani Eskandani, Ali Reza Zamani and H. Vaezi}

(Communicated by Nicolae Popa)

2000 Mathematics Subject Classification. Primary 46S40; Secondary 39B52, 39B82, 26E50, 46S50.

Keywords and phrases. Hyers-Ulam-Rassias stability, Fuzzy normed space, Additive mapping.

Abstract. In this paper, we investigate the generalized Hyers- UlamRassias stability of the functional equation

$$
\sum_{i=1}^{m} f\left(m x_{i}+\sum_{j=1, j \neq i}^{m} x_{j}\right)+f\left(\sum_{i=1}^{m} x_{i}\right)=2 f\left(\sum_{i=1}^{m} m x_{i}\right)
$$

in fuzzy Banach spaces and some applications of our results in the stability of above mapping from a normed space to a Banach space will be exhibited.

\section{Introduction and preliminaries}

In 1940, S.M. Ulam [27] asked the fundamental question for the stability of approximate homomorphisms. In 1941, D. H. Hyers [7] provided a partial solution to Ulam's problem for mappings between Banach spaces. This result was generalized by Aoki [2] for additive mappings and by Th. M. Rassias [26] for linear mappings by considering an unbounded Cauchy difference. The paper of Th. M. Rassias [26] has provided a lot of influence in the 
development of what we now call generalized Hyers-Ulam-Rassias stability of functional equations. In 1994, a generalization of Th. M. Rassias' Theorem was obtained by Găvruta [5]. During the last two decades, a number of papers and research monographs have been published on various generalizations and applications of the generalized Hyers-Ulam stability to a number of functional equations and mappings (see [6], [9], [15]- [25]). We also refer the readers to the books [1], [3], [8] and [10].

We recall some basic facts concerning fuzzy normed space.

Let $X$ be a real linear space. A function $N: X \times \mathbb{R} \longrightarrow[0,1]$ (socalled fuzzy subset) is said to be a fuzzy norm on $X$ if for all $x, y \in X$ and all $s, t \in \mathbb{R}$,

$(N 1) \quad N(x, t)=0$ for $t \leq 0$;

(N2) $\quad x=0$ if and only if $N(x, t)=1$ for all $t>0$;

(N3) $\quad N(s x, t)=N(x, t /|s|)$ if $s \neq 0$;

(N4) $\quad N(x+y, s+t) \geq \min \{N(x, s), N(y, t)\}$

$(N 5) N(x,$.$) is a non-decreasing function on \mathbb{R}$ and

$$
\lim _{t \rightarrow \infty} N(x, t)=1
$$

The pair $(X, N)$ is called a fuzzy normed linear space.

Example 1.1. Let $(X,\|\cdot\|)$ be a normed linear space. Then

$$
N(x, t)=\left\{\begin{array}{lc}
\frac{t}{t+\|x\|} & t>0, x \in X \\
0 & t \leq 0, x \in X
\end{array}\right.
$$

is a fuzzy norm on $X$.

Example 1.2. Let $(X,\|\cdot\|)$ be a normed linear space. Then

$$
N(x, t)=\left\{\begin{array}{cc}
0 & t \leq\|x\|, x \in X \\
1 & t>\|x\|, x \in X
\end{array}\right.
$$

is a fuzzy norm on $X$.

Let $(X, N)$ be a fuzzy normed linear space. Let $\left\{x_{n}\right\}$ be a sequence in $\mathrm{X}$. Then $\left\{x_{n}\right\}$ is said to be convergent if there exists $x \in X$ such that

$$
\lim _{n \rightarrow \infty} N\left(x_{n}-x, t\right)=1
$$

for all $t>0$. In that case, $x$ is called the limit of the sequence $\left\{x_{n}\right\}$, and we write $N-\lim _{n \rightarrow \infty} x_{n}=x$. 
A sequence $\left\{x_{n}\right\}$ in a fuzzy normed space $(X, N)$ is called Cauchy if for each $\epsilon>0$ and each $\delta>0$, one can find some $n_{0}$ such that

$$
N\left(x_{m}-x_{n}, \delta\right)>1-\epsilon
$$

for all $n, m \geq n_{0}$.

It is known that every convergent sequence in a fuzzy normed space is Cauchy. If in a fuzzy normed space, each Cauchy sequence is convergent, then the fuzzy norm is said to be complete and the fuzzy normed space is called a fuzzy Banach space.

Stability of Cauchy, Jensen, quadratic and cubic function equation in fuzzy normed spaces have been investigated in [11][14]. C. Park [20] has proved the generalized Hyers-Ulam stability of a functional equation associated with inner product spaces in fuzzy Banach spaces.

In this paper, we investigate the generalized Hyers- Ulam-Rassias stability of the functional equation

$$
\sum_{i=1}^{m} f\left(m x_{i}+\sum_{j=1, j \neq i}^{m} x_{j}\right)+f\left(\sum_{i=1}^{m} x_{i}\right)=2 f\left(\sum_{i=1}^{m} m x_{i}\right)
$$

in fuzzy Banach spaces, where $m$ is a fixed positive integer with $m \geq 2$.

\section{Fuzzy Stability of functional equation (1)}

Hereafter, unless otherwise stated, we will assume that $X$ is real vectors space, $(Y, N)$ is a complete fuzzy normed space and $m$ is a fixed integer greater than 1. For convenience, we use the following abbreviation for a given mapping $f$

$$
D f\left(x_{1}, \ldots, x_{m}\right):=\sum_{i=1}^{m} f\left(m x_{i}+\sum_{j=1, j \neq i}^{m} x_{j}\right)+f\left(\sum_{i=1}^{m} x_{i}\right)-2 f\left(\sum_{i=1}^{m} m x_{i}\right) .
$$

We will use the following lemma [4]:

Lemma 2.1. A mapping $f: X \rightarrow Y$ satisfies (1) if and only if it is additive.

Theorem 2.2. Let $\left(Z, N^{\prime}\right)$ be a fuzzy normed space and $\varphi$ : $\underbrace{X \times X \times \ldots \times X}_{m \text {-times }} \rightarrow Z$ be a mapping such that $\varphi(0, \ldots, \underbrace{m x}_{j t h}, . ., 0)=$ 
208 Fuzzy approximation of an additive functional equation

$\alpha \varphi(0, \ldots, \underbrace{x}_{j t h}, . ., 0)$ for some $\alpha$ with $0<\alpha<m$. Asumme that $f: X \longrightarrow Y$ be mapping such that

$$
N\left(D f\left(x_{1}, \ldots, x_{m}\right), t\right) \geq N^{\prime}\left(\varphi\left(x_{1}, \ldots, x_{m}\right), t\right)
$$

for all $x_{i} \in X \quad(1 \leq i \leq m)$ and all positive real number $t$. Then there is a unique additive mapping $T: X \rightarrow Y$ such that $T(x)=$ $N-\lim _{n \rightarrow \infty} f\left(m^{n} x\right) / m^{n}$ and

$$
N(T(x)-f(x), t) \geq N^{\prime}\left(\varphi(0, \ldots, \underbrace{x}_{j t h}, . ., 0), \frac{m-\alpha}{2 \alpha} t\right)
$$

for all $x \in X$.

Proof. Letting $x_{j}=x$ and $x_{i}=0$ for all $(1 \leq i \leq m)$ with $i \neq j$ in (2), we get

$$
N(f(m x)-m f(x), t) \geq N^{\prime}(\varphi(0, \ldots, \underbrace{m x}_{j t h}, . ., 0), t)
$$

for all $x \in X$ and all positive real number $t$. Replacing $x$ by $m^{n} x$ in (3) , we get

$$
N\left(f\left(m^{n+1} x\right)-m f\left(m^{n} x\right), t\right) \geq N^{\prime}\left(\varphi(0, \ldots, \underbrace{m^{n+1} x}_{j t h}, . ., 0), t\right) .
$$

Thus

$$
N\left(\frac{f\left(m^{n+1} x\right)}{m^{n+1}}-\frac{f\left(m^{n} x\right)}{m^{n}}, \frac{t}{m^{n+1}}\right) \geq N^{\prime}\left(\varphi(0, \ldots, \underbrace{x}_{j t h}, . ., 0), \frac{t}{\alpha^{n+1}}\right)
$$

for all $x \in X$ and all positive real number $t$. Hence

(6)

$$
\begin{aligned}
& N\left(\frac{1}{m^{n+1}} f\left(m^{n+1} x\right)-\frac{1}{m^{k}} f\left(m^{k} x\right), \sum_{i=k}^{n} \frac{\alpha^{i+1}}{m^{i+1}} t\right) \\
& \quad=N\left(\sum_{i=k}^{n} \frac{1}{m^{i+1}} f\left(m^{i+1} x\right)-\frac{1}{m^{i}} f\left(m^{i} x\right), \sum_{i=k}^{n} \frac{\alpha^{i+1}}{m^{i+1}} t\right) \\
& \quad \geq \min \bigcup_{i=1}^{n}\left\{N\left(\frac{1}{m^{i+1}} f\left(m^{i+1} x\right)-\frac{1}{m^{i}} f\left(m^{i} x\right), \frac{\alpha^{i+1}}{m^{i+1}} t\right)\right\} \\
& \quad \geq N^{\prime}(\varphi(0, \ldots, \underbrace{x}_{j t h}, . ., 0), t) .
\end{aligned}
$$


Let $\epsilon>0$ and $\delta>0$ be given. Since $\lim _{t \rightarrow \infty} N^{\prime}(\varphi(0, \ldots, \underbrace{x}_{j t h}, . ., 0), t)=1$, there is some $t_{0}>0$ such that $N^{\prime}\left(\varphi(0, \ldots, \underbrace{x}_{j t h}, . ., 0), t_{0}\right)>1-\epsilon$. Since $\sum_{n=0}^{\infty} \frac{\alpha^{n}}{m^{n}} t_{0}<\infty$, there is some $n_{0} \in N$ such that $\sum_{i=k}^{n} \frac{\alpha^{i}}{m^{i}} t_{0}<\frac{m \delta}{\alpha}$ for all $n>k \geq n_{0}$. It follows that

$$
\begin{aligned}
N & \left(\frac{1}{m^{n+1}} f\left(m^{n+1} x\right)-\frac{1}{m^{k}} f\left(m^{k} x\right), \delta\right) \\
& \geq N\left(\frac{1}{m^{n+1}} f\left(m^{n+1} x\right)-\frac{1}{m^{k}} f\left(m^{k} x\right), \sum_{i=k}^{n} \frac{\alpha^{i+1}}{m^{i+1}} t_{0}\right) \\
& \geq N^{\prime}\left(\varphi(0, \ldots, \underbrace{x}_{j t h}, . ., 0), t_{0}\right)>1-\epsilon
\end{aligned}
$$

for all $x \in X$ and all non-negative integers $n$ and $k$ with $n>k \geq n_{0}$. Therefore, the sequence $\left\{\frac{1}{m^{n}} f\left(m^{n} x\right)\right\}$ is a Cauchy sequence in $(Y, N)$ for all $x \in X$. Since $(Y, N)$ is complete, the sequence $\left\{\frac{1}{m^{n}} f\left(m^{n} x\right)\right\}$ converges in $Y$ for all $x \in X$. So one can define the mapping $T: X \rightarrow Y$ by

$$
T(x):=N-\lim _{n \rightarrow \infty} \frac{1}{m^{n}} f\left(m^{n} x\right)
$$

for all $x \in X$. Now, we show that $T$ is additive mapping. It follows from (2) and (8) that

$$
\begin{aligned}
N\left(D T\left(x_{1}, \ldots, x_{m}\right), t\right) & =\lim _{n \rightarrow \infty} N\left(\frac{D f\left(m^{n} x_{1}, \ldots, m^{n} x_{m}\right)}{m^{n}}, t\right) \\
& \geq \lim _{n \rightarrow \infty} N^{\prime}\left(\frac{\varphi\left(m^{n} x_{1}, \ldots, m^{n} x_{m}\right)}{m^{n}}, t\right) \\
& =\lim _{n \rightarrow \infty} N^{\prime}\left(\varphi\left(x_{1}, \ldots, x_{m}\right), \frac{m^{n}}{\alpha^{n}} t\right) \\
& =1
\end{aligned}
$$

for all $x_{1}, \cdots, x_{m} \in X$ and all positive real numbers $t$. So $N\left(D T\left(x_{1}, \ldots, x_{m}\right), t\right)=1$ for all $x_{1}, \cdots, x_{m} \in X$ and all positive real numbers $t$. Therefor $D T\left(x_{1}, \ldots, x_{m}\right)=0$. By Lemma (2.1), the mapping $T: X \rightarrow Y$ is additive. Moreover, if we put $k=0$ in (6), we observe that

$$
N\left(\frac{1}{m^{n+1}} f\left(m^{n+1} x\right)-f(x), \sum_{i=0}^{n} \frac{\alpha_{j}^{i+1}}{m^{i+1}} t\right) \geq N^{\prime}(\varphi(0, \ldots, \underbrace{x}_{j t h}, . ., 0), t) .
$$


210 Fuzzy approximation of an additive functional equation

Therefore,

(9) $N\left(\frac{1}{m^{n+1}} f\left(m^{n+1} x\right)-f(x), t\right) \geq N^{\prime}\left(\varphi(0, \ldots, \underbrace{x}_{j t h}, . ., 0), \frac{t}{\sum_{i=0}^{n} \frac{\alpha_{1}^{i+1}}{m^{i+1}}}\right)$

It follows from (9) that, for large enough $n$,

$$
\begin{aligned}
& N(T(x)-f(x), t) \\
& \geq \min \left\{N\left(\frac{1}{m^{n+1}} f\left(m^{n+1} x\right)-f(x), \frac{t}{2}\right), N\left(T(x)-\frac{1}{m^{n+1}} f\left(m^{n+1} x\right), \frac{t}{2}\right)\right\} \\
& \geq N^{\prime}\left(\varphi(0, \ldots, \underbrace{x}_{j t h}, . ., 0), \frac{t}{2 \sum_{i=0}^{n} \frac{\alpha_{j}^{i+1}}{m^{i+1}}}\right) \\
& \geq N^{\prime}\left(\varphi(0, \ldots, \underbrace{x}_{j t h}, . ., 0), \frac{m-\alpha}{2 \alpha} t\right) .
\end{aligned}
$$

Now, we show that $T$ is unique. Let $T^{\prime}$ be another additive mapping from $X$ into $Y$, which satisfies the required inequality. Then for each $x \in X$ and $t>0$, we have

$$
\begin{aligned}
N\left(T(x)-T^{\prime}(x), t\right) & \geq \min \left\{N\left(T(x)-f(x), \frac{t}{2}\right), N\left(f(x)-T^{\prime}(x), \frac{t}{2}\right)\right\} \\
& \geq N^{\prime}\left(\varphi(0, \ldots, \underbrace{x}_{j t h}, . ., 0), \frac{m-\alpha}{4 \alpha} t\right) .
\end{aligned}
$$

So

$$
\begin{aligned}
N\left(T(x)-T^{\prime}(x), t\right) & =N\left(\frac{T\left(m^{n} x\right)}{m^{n}}-\frac{T^{\prime}\left(m^{n} x\right)}{m^{n}}, t\right) \\
& \geq N^{\prime}\left(\frac{\alpha^{n}}{m^{n}} \varphi(0, \ldots, \underbrace{x}_{j t h}, . ., 0), \frac{m-\alpha}{4 \alpha} t\right) .
\end{aligned}
$$

Hence, the right hand side of the above inequality tends to 1 as $n \longrightarrow \infty$. It follows that $T(x)=T^{\prime}(x)$ for all $x \in X$.

Theorem 2.3. Let $\left(Z, N^{\prime}\right)$ be a fuzzy normed space and $\Phi$ : $\underbrace{X \times X \times \ldots \times X}_{m \text {-times }} \rightarrow Z$ be a mapping such that $\Phi(0, \ldots, \underbrace{m x}_{j t h}, . ., 0)=$ $\alpha \Phi(0, \ldots, \underbrace{x}_{j t h}, . ., 0)$ for some $\alpha$ with $\alpha>m$. Assume that $f: X \longrightarrow Y$ be mapping such that

$$
N\left(D f\left(x_{1}, \ldots, x_{m}\right), t\right) \geq N^{\prime}\left(\Phi\left(x_{1}, \ldots, x_{m}\right), t\right)
$$

for all $x_{i} \in X \quad(1 \leq i \leq m)$ and all positive real number $t$. Then there is a unique additive mapping $T: X \rightarrow Y$ such that $T(x)=$ 
$N-\lim _{n \rightarrow \infty} m^{n} f\left(\frac{x}{m^{n}}\right)$ and for all $x \in X$

$$
N(T(x)-f(x), t) \geq N^{\prime}\left(\Phi(0, \ldots, \underbrace{x}_{j t h}, . ., 0), \frac{\alpha-m}{2 \alpha} t\right) .
$$

Proof. Similarly to the proof of Theorem 2.2, we have

$$
N(f(m x)-m f(x), t) \geq N^{\prime}(\Phi(0, \ldots, \underbrace{m x}_{j t h}, . ., 0), t)
$$

for all $x \in X$ and all positive real number $t$. Replacing $x$ by $\frac{x}{m^{n+1}}$ in (11), we get

$$
N\left(f\left(\frac{x}{m^{n}}\right)-m f\left(\frac{x}{m^{n+1}}\right), t\right) \geq N^{\prime}\left(\Phi(0, \ldots, \underbrace{\frac{x}{m^{n}}}_{j t h}, . ., 0), t\right) .
$$

Thus

$$
N\left(m^{n} f\left(\frac{x}{m^{n}}\right)-m^{n+1} f\left(\frac{x}{m^{n+1}}\right), m^{n} t\right) \geq N^{\prime}\left(\Phi(0, \ldots, \underbrace{x}_{j t h}, . ., 0), \alpha^{n} t\right)
$$

for all $x \in X$ and all positive real number $t$. Hence

$$
\begin{aligned}
N\left(m^{n+1} f\left(\frac{x}{m^{n+1}}\right)-\right. & \left.m^{k} f\left(\frac{x}{m^{k}}\right), \sum_{i=k}^{n} \frac{m^{i}}{\alpha^{i}} t\right) \geq N\left(\sum_{i=k}^{n} m^{i+1} f\left(\frac{x}{m^{i+1}}\right)-m^{i} f\left(\frac{x}{m^{i}}\right), \sum_{i=k}^{n} \frac{m^{i}}{\alpha^{i}} t\right) \\
& \geq \min \bigcup_{i=1}^{m}\left\{N\left(m^{i+1} f\left(\frac{x}{m^{i+1}}\right)-m^{i} f\left(\frac{x}{m^{i}}\right), \frac{m^{i}}{\alpha^{i}} t\right)\right\} \\
& \geq N^{\prime}(\Phi(0, \ldots, \underbrace{x}_{j t h}, . ., 0), t) .
\end{aligned}
$$

Let $\epsilon>0$ and $\delta>0$ be given. Since $\lim _{t \rightarrow \infty} N^{\prime}(\Phi(0, \ldots, \underbrace{x}_{j t h}, . ., 0), t)=1$, there is some $t_{0}>0$ such that $N^{\prime}\left(\Phi(0, \ldots, \underbrace{x}_{j t h}, . ., 0), t_{0}\right)>1-\epsilon$. Since $\sum_{n=0}^{\infty} \frac{m^{n}}{\alpha^{n}} t_{0}<\infty$, there is some $n_{0} \in N$ such that $\sum_{i=k}^{n} \frac{m^{i}}{\alpha^{i}} t_{0}<\delta$ for all $n>k \geq n_{0}$. It follows from (14) that

$$
\begin{aligned}
N\left(m^{n+1} f\left(\frac{x}{m^{n+1}}\right)-m^{k} f\left(\frac{x}{m^{k}}\right), \delta\right) & \geq N\left(m^{n+1} f\left(\frac{x}{m^{n+1}}\right)-m^{k} f\left(\frac{x}{m^{k}}\right), \sum_{i=k}^{n} \frac{m^{i}}{\alpha_{j}^{i}} t_{0}\right) \\
& \geq N^{\prime}\left(\Phi(0, \ldots, \underbrace{x}_{j t h}, . ., 0), t_{0}\right)>1-\epsilon
\end{aligned}
$$


212 Fuzzy approximation of an additive functional equation

for all $x \in X$ and all non-negative integers $n$ and $k$ with $n>k \geq n_{0}$. Therefore, the sequence $\left\{m^{n} f\left(\frac{x}{m^{n}}\right)\right\}$ is a Cauchy sequence in $(Y, N)$ for all $x \in X$. Since $(Y, N)$ is complete, the sequence $\left\{m^{n} f\left(\frac{x}{m^{n}}\right)\right\}$ converges in $Y$ for all $x \in X$. So one can define the mapping $T: X \rightarrow Y$ by

$$
T(x):=N-\lim _{n \rightarrow \infty} m^{n} f\left(\frac{x}{m^{n}}\right), \quad x \in X .
$$

The rest of the proof is similar to the proof of Theorem 2.2

Corollary 2.4. Let $X$ be a normed space and $\left(Z, N^{\prime}\right)$ be a fuzzy normed space. Assume that $r_{i}(1 \leq i \leq m)$ be positive real numbers such that $r_{i}<1$ or $r_{i}>1(1 \leq i \leq m)$ and $f: X \longrightarrow Y$ be a mapping such that

$$
N\left(D f\left(x_{1}, \ldots, x_{m}\right), t\right) \geq N^{\prime}\left(\sum_{i=1}^{m}\left\|x_{i}\right\|^{r_{i}} z_{0}, t\right)
$$

for all $x_{i} \in X \quad(1 \leq i \leq m)$ and all positive real number $t$, where $z_{0}$ is a fixed vector of $Z$. Then there is a unique additive mapping $T: X \longrightarrow Y$ such that

$$
N(T(x)-f(x), t) \geq N^{\prime}\left(\|x\|^{r_{j}} z_{0},\left|\frac{1}{m^{r_{j}-1}}-1\right| \frac{t}{2}\right)
$$

for all $x \in X$.

Corollary 2.5. Let $X$ be a normed space and $\left(Z, N^{\prime}\right)$ be a fuzzy normed space. Assume that $r_{i}(1 \leq i \leq m)$ be positive real numbers such that $r_{1}+\ldots+r_{m} \neq 1$ and $f: X \longrightarrow Y$ be a mapping such that

$$
N\left(D f\left(x_{1}, \ldots, x_{m}\right), t\right) \geq N^{\prime}\left(\prod_{i=1}^{m}\left\|x_{i}\right\|^{r_{i}} z_{0}, t\right)
$$

for all $x_{i} \in X \quad(1 \leq i \leq m)$ and all positive real number $t$, where $z_{0}$ is a fixed vector of $Z$. Then $f: X \longrightarrow Y$ is additive.

Proof. Letting $x_{1}=m x$ and $x_{i}=0$ for all $i(2 \leq i \leq m)$ in (18), we get

$$
N(f(m x)-m f(x), t)=1
$$

for all $x \in X$ and all positive real number $t$. So

$$
f(m x)=m f(x)
$$

for all $x \in X$. Hence the result follows by Theorems 2.2 and 2.3. 


\section{Application of fuzzy stability to the stability of Ex. (1) in normed spaces}

Hereafter, we will assume that $X$ is a normed space and $Y$ is a Banach space. Let $Z=\mathbb{R}$. As noted in Example 1.1

$$
N^{\prime}(z, t)= \begin{cases}\frac{t}{t+|z|} & t>0, z \in Z \\ 0 & t \leq 0, z \in Z\end{cases}
$$

defines a fuzzy norm on $Z$ and

$$
N(y, t)= \begin{cases}\frac{t}{t+\|y\|} & t>0, y \in Y \\ 0 & t \leq 0, y \in Y\end{cases}
$$

defines a fuzzy norm on $Y$. By using Corollaries 2.4 and 2.5, one can obtain the following corollaries.

Corollary 3.1. Let $\theta, r_{i}(1 \leq i \leq m)$ be positive real numbers such that $r_{i}<1$ or $r_{i}>1(1 \leq i \leq m)$. Suppose that a mapping $f: X \rightarrow Y$ satisfies the inequality $\left\|D f\left(x_{1}, \ldots, x_{m}\right)\right\| \leq \theta \Sigma_{i=1}^{m}\left\|x_{i}\right\|^{r_{i}}$ for all $x_{i} \in X$ $(1 \leq i \leq m)$. Then there exists a unique additive mapping $T: X \rightarrow Y$ satisfying

$$
\|f(x)-T(x)\| \leq \frac{2 m^{r_{j}} \theta}{\left|m-m^{r_{j}}\right|}\|x\|^{r_{j}}, \quad x \in X .
$$

Corollary 3.2. Let $\theta, r_{i}(1 \leq i \leq m)$ be positive real numbers such that $r_{1}+\ldots+r_{m} \neq 1$. Suppose that a mapping $f: X \rightarrow Y$ satisfies the inequality

$$
\left\|D f\left(x_{1}, \ldots, x_{m}\right)\right\| \leq \theta \prod_{i=1}^{m}\left\|x_{j}\right\|^{r_{j}}
$$

for all $x_{i} \in X \quad(1 \leq j \leq m)$. Then the mapping $f: X \rightarrow Y$ is additive.

\section{References}

[1] J. Aczél and J. Dhombres, Functional Equations in Several Variables, Cambridge University Press, 1989.

[2] T. Aoki, On the stability of the linear transformation in Banach spaces, J. Math. Soc. Japan, 2 (1950) 64-66. 
214 Fuzzy approximation of an additive functional equation

[3] S. Czerwik, Functional Equations and Inequalities in Several Variables, World Scientific, New Jersey, London, Singapore, Hong Kong, 2002.

[4] G.Z. Eskandani, On the Hyers-Ulam-Rassias stability of an additive functional equation in quasi-Banach spaces, J. Math. Anal. Appl., 345 (2008) 405-409.

[5] P. Găvruta, A generalization of the Hyers-Ulam-Rassias stability of approximately additive mappings, J. Math. Anal. Appl., 184 (1994), 431-436.

[6] P. Găvruta, On a problem of G. Isac and Th. M. Rassias concerning the stability of mappings, J. Math. Anal. Appl., 261 (2001), 543-553.

[7] D. H. Hyers, On the stability of the linear functional equation, Proc. Natl. Acad. Sci., 27 (1941), 222-224.

[8] D.H. Hyers, G. Isac and Th.M. Rassias, Stability of Functional Equations in Several Variables, Birkhäuser, Basel, 1998.

[9] K. Jun and Y. Lee, On the Hyers-Ulam-Rassias stability of a Pexiderized quadratic inequality, Math. Inequal. Appl., 4 (2001), 93-118.

[10] S. M. Jung, Hyers-Ulam-Rassias Stability of Functional Equations in Mathimatical Analysis, Hadronic Press, Palm Harbor, 2001.

[11] A.K. Mirmostafaee and M.S. Moslehian, Fuzzy versions of Hyers-Ulam-Rassias theorem, Fuzzy Sets and Systems, 159 (2008) 720-729.

[12] A.K. Mirmostafaee and M.S. Moslehian, Fuzzy approximately cubic mappings, Information Sciences, 178 (2008) 3791-3798.

[13] A.K. Mirmostafaee, M. Mirzavaziri and M.S. Moslehian, Fuzzy Almost Quadratic Functions, Result.Math. 52 (2008), 161-177.

[14] A.K. Mirmostafaee, M. Mirzavaziri and M.S. Moslehian, Fuzzy stability of the Jensen functional equation, Fuzzy Sets and Systems, 159 (2008), 730-738.

[15] M. Mirzavaziri and M.S. Moslehian, A fixed point approach to stability of a quadratic equation, Bull. Braz. Math. Soc., 37 (2006), 361-376.

[16] M. S. Moslehian, On the orthogonal stability of the Pexiderized quadratic equation, J. Differ. Equations. Appl., 11, No.11(2005), 999-1004. 
[17] A. Najati and G.Z. Eskandani, Stability of a mixed additive and cubic functional equation in quasi-Banach spaces, J. Math. Anal. Appl., 342 (2008), 1318-1331.

[18] C. Park, Homomorphisms between Lie $J C^{*}$-algebras and Cauchy-Rassias stability of Lie $J C^{*}$-algebra derivations, J. Lie Theory, 15 (2005), 393-414.

[19] C. Park, Homomorphisms between Poisson JC*-algebras, Bull. Braz. Math. Soc., 36 (2005), 79-97.

[20] C. Park, Fuzzy stability of a functional equation associated with inner product spaces, Fuzzy Sets and Systems, 160 (2009), 1632-1642.

[21] J.M. Rassias, On approximation of approximately linear mappings by linear mappings, J. Funct. Anal., 46 (1982) 126130.

[22] J.M. Rassias, On approximation of approximately linear mappings by linear mappings, Bull. Sci. Math., 108 (1984), 445-446.

[23] J.M.Rassias, Solution of a problem of Ulam, J. Approx. Theory, 57(1989), 268-273.

[24] Th.M. Rassias, On a modified Hyers-Ulam sequence, J. Math. Anal. Appl., 158 (1991), 106-113.

[25] Th.M. Rassias, On the stability of functional equations and a problem of Ulam, Acta Appl. Math., 62 (2000), 23-130.

[26] Th.M. Rassias, On the stability of the linear mapping in Banach spaces, Proc. Amer. Math. Soc., 72 (1978), 297-300.

[27] S.M. Ulam, A Collection of the Mathematical Problems, Interscience Publ., New York, 1960.

Faculty of Mathematical Sciences

University of Tabriz, Tabriz, Iran

(E-mail : zamani@tabrizu.ac.ir)

Faculty of Science

Department of Mathematics

Tabriz Mathematics House,Tabriz,Iran

(E-mail : zamani.alireza@gmail.com)

Faculty of Mathematical Sciences

University of Tabriz,Tabriz, Iran

(E-mail : hvaezi@tabrizu.ac.ir)

(Received : January 2010) 


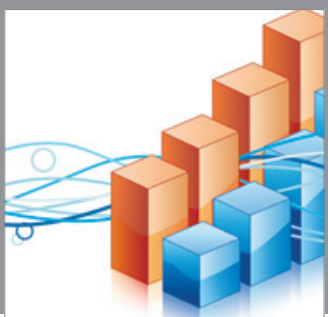

Advances in

Operations Research

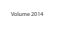

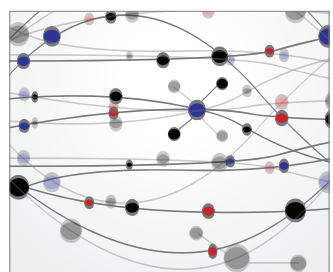

\section{The Scientific} World Journal
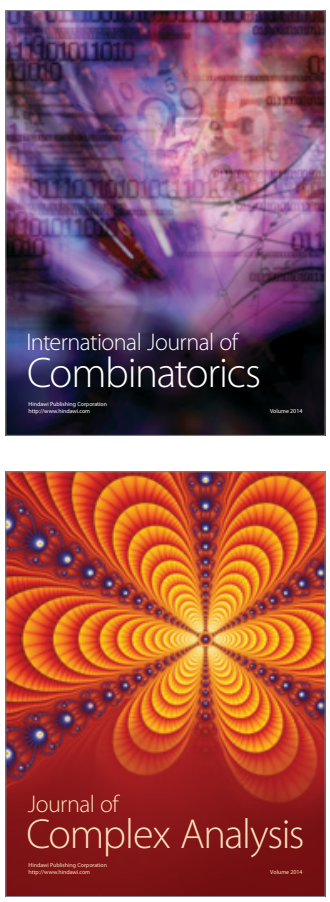

International Journal of

Mathematics and

Mathematical

Sciences
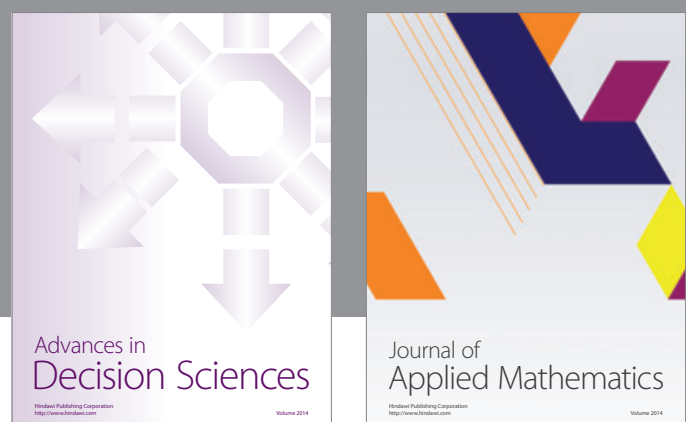

Journal of

Applied Mathematics
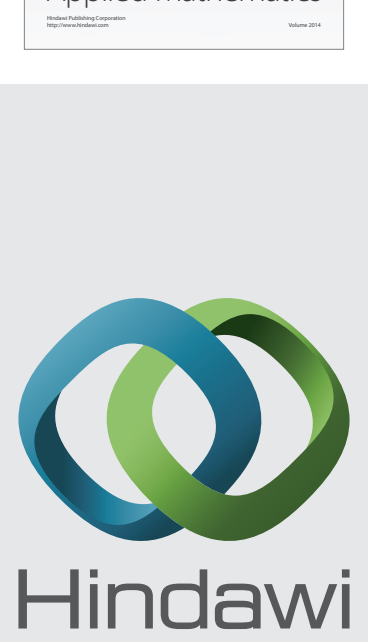

Submit your manuscripts at http://www.hindawi.com
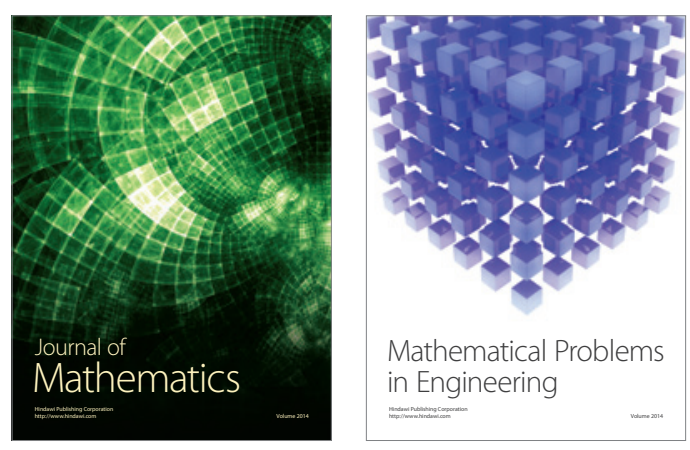

Mathematical Problems in Engineering
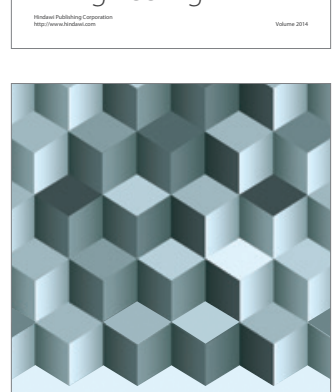

Journal of

Function Spaces
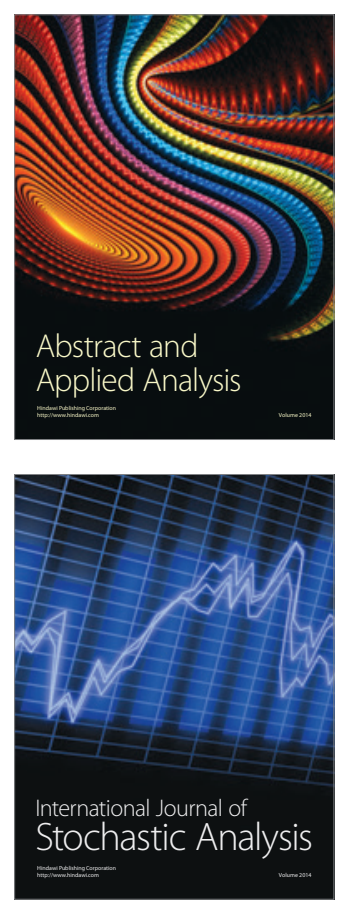

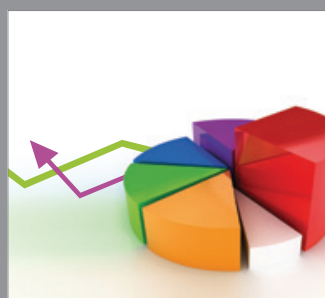

ournal of

Probability and Statistics

Promensencen
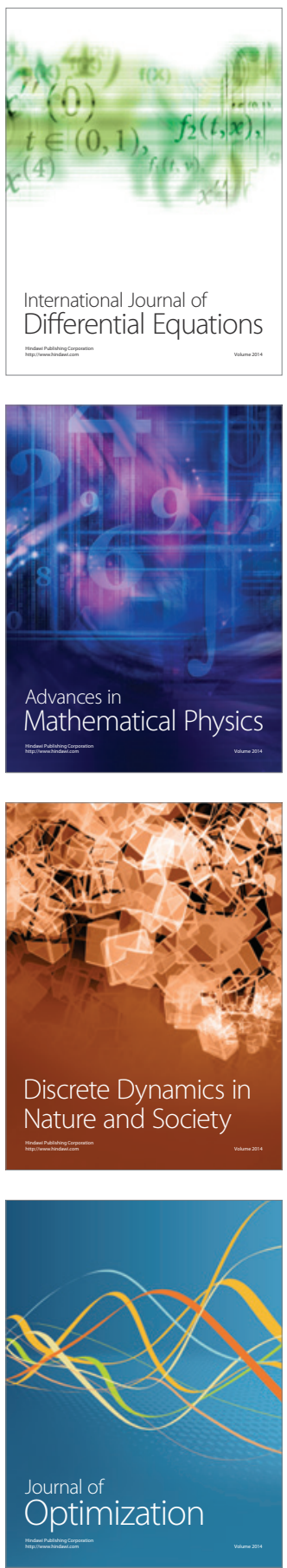